\title{
Age-specific and sex-specific weight gain norms to monitor antiretroviral therapy in children in low-income and middle-income countries
}

\author{
Marcel Yotebieng ${ }^{a, b}$, Tammy Meyers ${ }^{c}$, Frieda Behets ${ }^{d}$, \\ Mary-Ann Davies ${ }^{\mathrm{e}}$, Olivia Keiser ${ }^{\mathrm{f}}$, Kapella Zacharia Ngonyanig, \\ Rita E. Lyamuyah ${ }^{\text {h }}$ Azar Kariminiai, Rawiwan Hansudewechakuli, \\ Valeriane Leroy $^{k, I}$, Sikiratou Koumakpai ${ }^{m}$, Jamie Newman ${ }^{n}$ and \\ Annelies Van Rie ${ }^{\mathbf{b}}$
}

\begin{abstract}
Background: Viral load and CD4\% are often not available in resource-limited settings for monitoring children's responses to antiretroviral therapy (ART). We aimed to construct normative curves for weight gain at 6, 12, 18, and 24 months following initiation of ART in children, and to assess the association between poor weight gain and subsequent responses to ART.

Design: Analysis of data from HIV-infected children younger than 10 years old from African and Asian clinics participating in the International epidemiologic Databases to Evaluate AIDS.

Methods: The generalized additive model for location, scale, and shape was used to construct normative percentile curves for weight gain at 6, 12, 18, and 24 months following ART initiation. Cox proportional models were used to assess the association between lower percentiles ( $<50$ th) of weight gain distribution at the different time points and subsequent death, virological suppression, and virological failure.

Results: Among 7173 children from five regions of the world, $45 \%$ were underweight at baseline. Weight gain below the 50th percentile at 6, 12,18, and 24 months of ART was associated with increased risk of death, independent of baseline characteristics. Poor weight gain was not associated with increased hazards of virological suppression or virological failure.

Conclusion: Monitoring weight gain on ART using age-specific and sex-specific normative curves specifically developed for HIV-infected children on ART is a simple, rapid, sustainable tool that can aid in the identification of children who are at increased risk of death in the first year of ART.
\end{abstract}

(C) 2014 Wolters Kluwer Health | Lippincott Williams \& Wilkins

AIDS 2015, 29:101-109

Keywords: antiretroviral therapy monitoring, $\mathrm{CD}^{+}$, children, HIV, low-

income/middle-income countries, viral load, weight

\begin{abstract}
${ }^{a}$ Division of Epidemiology, College of Public Health, The Ohio State University, Columbus, Ohio, ${ }^{b}$ Department of Epidemiology, The University of North Carolina at Chapel Hill, Chapel Hill, North Carolina, USA, ${ }^{\mathrm{C}}$ Department of Pediatrics, Faculty of Health Sciences, University of the Witwatersrand, Johannesburg, South Africa, ${ }^{\mathrm{d}}$ School of Medicine, The University of North Carolina at Chapel Hill, Chapel Hill, North Carolina, USA, 'School of Public Health and Family Medicine, University of Cape Town, Cape Town, South Africa, ${ }^{f}$ Institute of Social and Preventive Medicine, University of Bern, Switzerland, ' Hospital, Kibaha, ' Morogoro Regional Hospital, Morogoro, Tanzania, 'University of New South Wales, The Kirby Institute for Infection and Immunity in Society, Darlinghurst, New South Wales, Australia, 'ंChiangrai Prachanukroh Hospital, Chiangrai, Thailand, kInstitut de Santé Publique Epidemiologie et Développement, Université Bordeaux, 'Inserm, Centre Inserm U897 'Epidémiologie et Biostatistique', Bordeaux, France, ${ }^{\mathrm{m}}$ Centre National Hospitalier, Universitaire Hubert K. Maga, Cotonou, Bénin, and ${ }^{n}$ RTI International, Biostatistics and Epidemiology, Research Triangle Park, North Carolina, USA.

Correspondence to Marcel Yotebieng, MD, PhD, MPH, Division of Epidemiology, College of Public Health, The Ohio State University, 304 Cunz Hall, 1841 Neil Avenue, Columbus, OH 43210, USA.
\end{abstract}

Tel: +1 614688 2133; fax: +1 614688 3533; e-mail: myotebieng@cph.osu.edu

Received: 25 July 2014; revised: 24 September 2014; accepted: 30 September 2014. 


\section{Introduction}

More than three million children live with HIV worldwide, of whom more than $90 \%$ live in sub-Saharan Africa [1]. In the absence of antiretroviral therapy (ART), a third of children infected perinatally will not survive to their first birthday, and more than half will not survive to their second birthday [2]. Successful initiation of ART in children is followed by a rapid decline in viral load, a rebound in $\mathrm{CD}^{+}$cell count, a reduction in mortality, and a rapid gain in weight, especially in the first 6-12 months of ART [3-7].

In developed nations, routine laboratory tests (HIV viral load, $\mathrm{CD}^{+}$cell count) are performed every $3-4$ months to monitor patients with HIV receiving ART [8]. The measurement of viral load and to some extent that of $\mathrm{CD}^{+}$cell count requires expensive and sophisticated technologies that cannot always be easily transferred or sustained in resource-poor settings. Recent studies in adults showed that routine $\mathrm{CD} 4^{+}$monitoring had small but significant benefits over clinical monitoring, [9,10] and viral load monitoring had no significant additional benefit over $\mathrm{CD}^{+}{ }^{+}$monitoring [9]. Similar benefits of routine monitoring of $\mathrm{CD}^{+}$cell count were reported in the only such trial conducted in children so far [11]. In addition, this trial demonstrated that monitoring of weight gain on ART is a sensitive indicator of first-line treatment failure in African children [11], supporting the WHO recommendations that in settings in which viral load is unavailable, clinical parameters, particularly the improvement in growth, be used for monitoring ART, supported where possible with $\mathrm{CD}^{+}$cell count monitoring [12].

Contrary to viral load, which has a clear and simple target cut-point (below detection limit), cut-points for weight gain that correlate with subsequent treatment outcomes have not been clearly established. One important difficulty in establishing those references for children resides in the fact that changes in weight strongly depend on age and sex. Two age-stratified and sex-stratified normative percentiles curves are almost ubiquitously used in pediatric care: the WHO 'Road to Health' for attained weight-for-age [13], and the Fels Institute growth charts for growth velocity $[14,15]$. In a previous analysis [16] using data from a single clinic in Soweto, South Africa, we demonstrated that the WHO and the Fels Institute growth charts were not valid for use in children receiving ART. Furthermore, although the effectiveness of ART is the same in high-income, middle-income, and lowincome countries $[17,18]$, the prevalence of malnutrition and opportunistic infections at ART initiation varies by region, which could affect weight gain following ART initiation.

In this study, we aimed to construct international reference standards for gains in weight at $6,12,18$, and 24 months following ART initiation and identify the centile curves of weight gain that are correlated with subsequent treatment failure and death.

\section{Methods}

\section{Data and data sources}

Data for this analysis were provided by the International Epidemiologic Databases to Evaluate AIDS, a US National Institutes of Health initiative launched in 2005 to establish an international research consortium to address research questions not answerable by single cohorts. The initiative funds seven regional data centers of which five contributed data for this analysis: the AsiaPacific region which includes the Therapeutics Research, Education, and AIDS Training in Asia (TREAT Asia) HIV Observational Database and includes data from Cambodia, India, Indonesia, Malaysia, Thailand, and Vietnam; the West African Database on Antiretroviral Therapy Collaboration which includes cohorts from Benin, Burkina Faso, Côte d'Ivoire, Ghana, Mali, and Senegal; the Central African region with participating sites from Burundi, Cameroon, Democratic Republic of Congo, and Rwanda; the Eastern Africa regional data centers which combine data from cohorts in Kenya, Tanzania, and Uganda; and the Southern African region with data from Lesotho, Malawi, Mozambique, South Africa, Zambia, and Zimbabwe. Detailed descriptions of the database and the main clinical outcomes have been reported elsewhere $[19,20]$.

\section{Statistical analysis}

Assessing the homogeneity of weight gain over time by region

To assess the homogeneity of gains in weight, we computed the median weight gain from ART initiation for each 3-month interval through 24 months of ART and plotted the values for each of the five regions. The plotted curves were visually inspected and data were merged if the gains overtime appeared homogeneous across regions (parallel plots). A quantile regression model with weight as response variable and time, region, and the interaction terms between region and time as dependent variable was also used to formally assess whether the change in weight over time after ART initiation varies by region.

Construction of reference curves for weight gain at 6 , 12,18 , and 24 months after antiretroviral therapy initiation

We calculated weight gain at each of the $6,12,18$, and 24 months time points for each individual child. Response curves were obtained by smoothing measurements over the chronological age at time of measurement using locally weighted quadratic regression [21]. Estimates of weight gain were then obtained by subtracting the response curve estimates at each time point from the baseline (at ART initiation) estimates (see reference [16] 
for a detailed description of the method). For visits that fell within 3 months of the cut-point, the weight gain was adjusted simply by dividing the measured weight gain by the exact time interval since ART initiation and multiplying it by the corresponding time interval. For example, in a child whose closest weight to 6 months was measured at 5 months, the estimate of weight gain at 6 months was obtained by dividing the difference between the smoothed value of weight at 5 months and the value at ART initiation by 5 , and then multi-plying it by 6 .

To obtain the normative reference percentile curves, we used methods similar to those used by the WHO to construct recent international growth curves [13]. The 6, 12,18 , and 24 months estimates of weight gain were regressed on chronological age using the generalized additive model for location, scale, and shape, a method that requires a parametric distribution assumption for the response variable while allowing the modeling of the distribution parameter as nonparametric (smooth) functions of the explanatory variables [22]. For the response variable, we assumed a Box-Cox power exponential distribution with four parameters relating to location ( $\mu$, median), scale ( $\sigma$, coefficient of variation), skewness ( $v$, transformation for symmetry), and kurtosis $(\tau$, power exponential parameter), respectively [23]. To specify the model, the user must choose the number of degrees of freedom (df) to be used for each parameter. Starting with the simplest model that includes age and the fitting of $\mu$ and $\sigma$ curves while keeping the degree of freedom for $v$ and $\tau$ fixed at zero, we searched for $\operatorname{df}(\mu)$ and then $\operatorname{df}(\sigma)$ that minimized the global deviance as indicated by the generalized Akaike Information Criterion (with penalty 3 for each degree of freedom used). In the next step, using the $\operatorname{df}(\mu)$ and $\operatorname{df}(\sigma)$ selected in the previous, we sequentially searched for the $\operatorname{df}(v)$ and $\operatorname{df}(\tau)$ that minimized the global deviance. In the last step, Q statistic [24] and worm plots [25] were used to fine tune the selected $\operatorname{df}(\mu), \operatorname{df}(\sigma), \operatorname{df}(v)$, and $\operatorname{df}(\tau)[23]$. Because of the high variability of weight gain in children after the age of 10 years, only data from children younger than 10 years were used to facilitate model convergence.

Association of lower weight gain with subsequent response to antiretroviral therapy

Three outcomes were considered: time to death (survival), time to viral suppression (first viral load less than 400 copies $/ \mathrm{ml}$ after ART initiation), and time to virologic failure. The outcome of virologic failure occurred when a child met one of three conditions: a viral load measurement more than 1000 copies/ml after at least 1 year of ART, two consecutive viral load measurements more than 400 copies $/ \mathrm{ml}$ after initial virologic suppression, or failure to ever achieve virological suppression after at least 1 year of ART.
For each of the three outcomes, separate Cox proportional hazard models were fitted for the 3rd, 10th, 25th, 33rd, and 50th centiles as predictors for each of the 6, 12, 18, and 24 months time points. Age at ART initiation ( $<2$ years, $2-4$ years, $5-9$ years), weight-forage $z$ score (WAZ) $(<-3 \mathrm{SD},-3 \mathrm{SD} \leq$ to $<-2 \mathrm{SD},-2 \mathrm{SD}$ $\leq$ to $<-1 \mathrm{SD}$, and $\geq-1 \mathrm{SD})[26]$, baseline CD4\% $(<15$, $15-25,>25 \%)$, an interaction term between WAZ and the centiles (in case the association differed by baseline WAZ), and year of ART initiation were included in the initial model for death. Baseline viral load $(\geq 5 \log$, $<5 \log$ copies $/ \mathrm{ml}$ ) was also included in the initial models for the two virological outcomes. Using a stepwise backward selection procedure and the Wald test, all covariates that did not contribute significantly to the fit of each multivariate model were dropped. The hazard ratio and $95 \%$ confidence interval (CI) from each of the final models are reported. All variables included in the model met the proportional hazards assumption formally evaluated using the Kolmogorov-type supremum test [27].

Analyses were done using SAS 9.2 (SAS Institute, Cary, North Carolina, USA). All tests were conducted using a two-sided 0.05 significance level, without correction for multiple comparisons (or uncertainty because of model selection). The study was approved by the Office of Human Research Ethics at the University of North Carolina at Chapel Hill.

\section{Results}

\section{Description of cohorts}

Of the 11802 HIV-infected children younger than 10 years of age in the combined dataset, 8628, 6825, 5241, and 3883, were on ART for at least 6, 12, 18, and 24 months, respectively. Of those children, 7173, 5029, 4288 , and 3072 had sufficient data to be included in the analysis at each time point. Half (3657 or 51\%) were from Southern Africa, 23\% from Eastern Africa, 13\% from Asia, 9\% from Western Africa, and 4\% from Central Africa (Table 1). The change in weight overtime following ART initiation was homogeneous across regions. All $P$ values for the four interaction terms between region and time were more than 0.20 (Figure 3 and Table 3 supplemental material, http://links.lww.com/QAD/A596).

Few (3.5\%) children initiated ART before 2004, the majority (78\%) initiated between 2005 and 2007, and the remainder (7\%) initiated in 2008 and 2009 (Table 1). Half $(52 \%)$ were male. At the time of ART initiation, 23\% were aged 1 year or younger, and $45 \%$ were underweight for age (WAZ $\leq-2 \mathrm{SD})$. Of the 5171 (72.1\%) children with pre-ART CD4\% available, $74 \%$ were severely immunosuppressed (CD4\% <15\%). Of the 2615 (36.5\%) children with pre-ART viral load, $64 \%$ had values at least $5 \log$ copies/ml. Children from the Eastern and Southern Africa regions were less likely to be underweight-for-age 


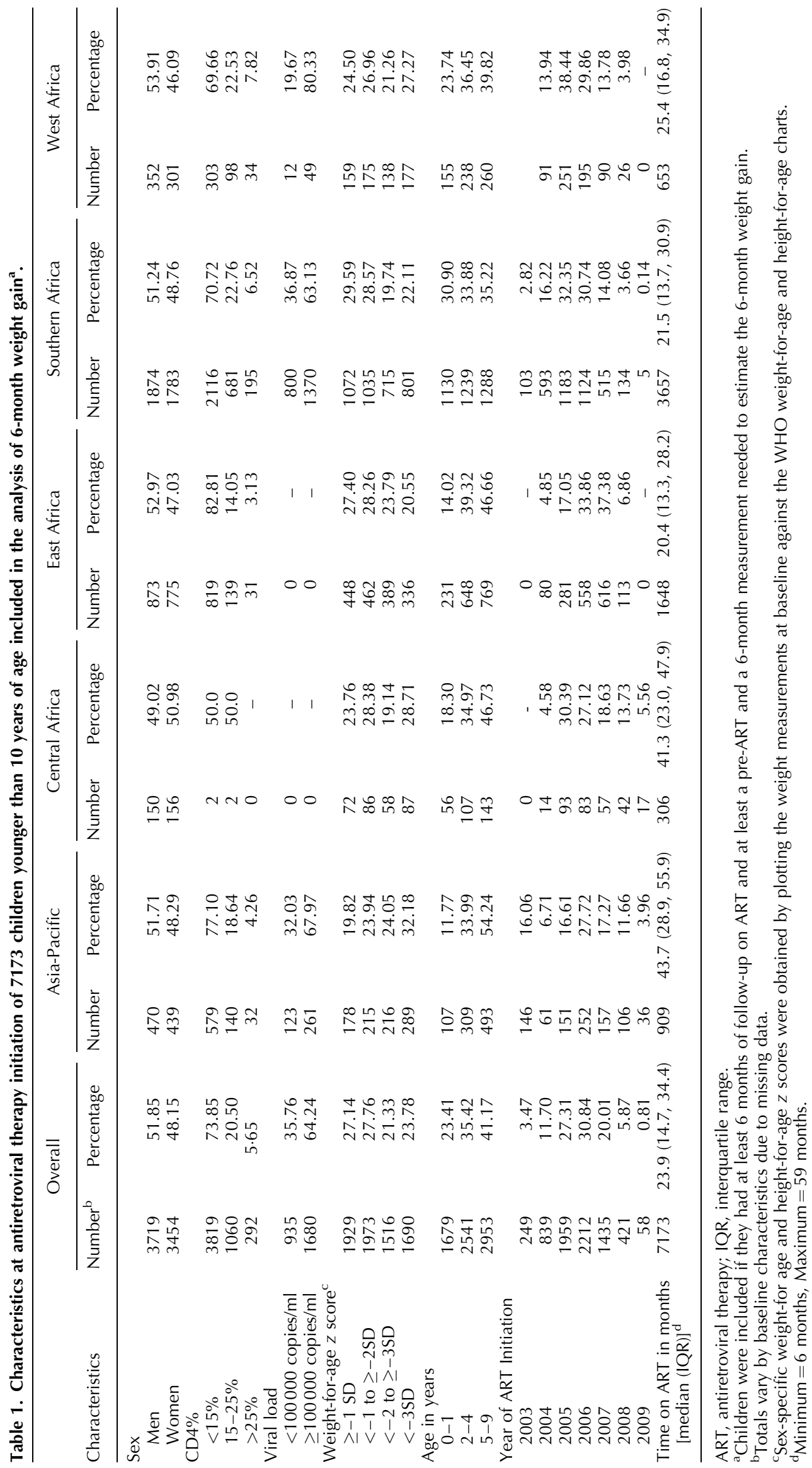


at ART initiation compared with those from other regions $(P<0.01)$.

The median duration of follow-up was 23.9 months following ART initiation. A total of 111 deaths were recorded, of which 68 (61.3\%) occurred between 6 and 12 months, 20 (18.0\%) between 12 and 18 months, 12 (10.8\%) between 18 and 24 months, and 11 (10.0\% after 24 months of ART (Table 5 supplemental material, http://links.lww.com/QAD/A596).

\section{Growth curves and distribution of $6,12,18$, and 24 months weight gain}

Figures 1 and 2 present the age-specific and sex-specific distributions of cumulative weight gained at 6,12, 18, and 24 months after ART initiation. For example, for a boy who started ART at the age of 6 months, at the $6,12,18$, and 24 months visits, to remain consistently above the 33rd percentile curves for weight gain, he must have cumulatively gained at least $2.04,3.42,4.52$, and $5.50 \mathrm{~kg}$, at the corresponding visit, irrespective of his initial weight (Tables 6a-9b, supplemental material, http://links.lww. com/QAD/A596).

\section{Association of poor postantiretroviral therapy weight gain $(<50$ th percentile) and subsequent survival, viral suppression and virologic failure}

Children with poor weight gain at 6 and 12 months of ART had a statistically higher hazard of death than those with good weight gain (Table 2). After adjustment for WAZ at ART initiation, the hazard ratios comparing children below the 33rd percentile of weight gain with (a)
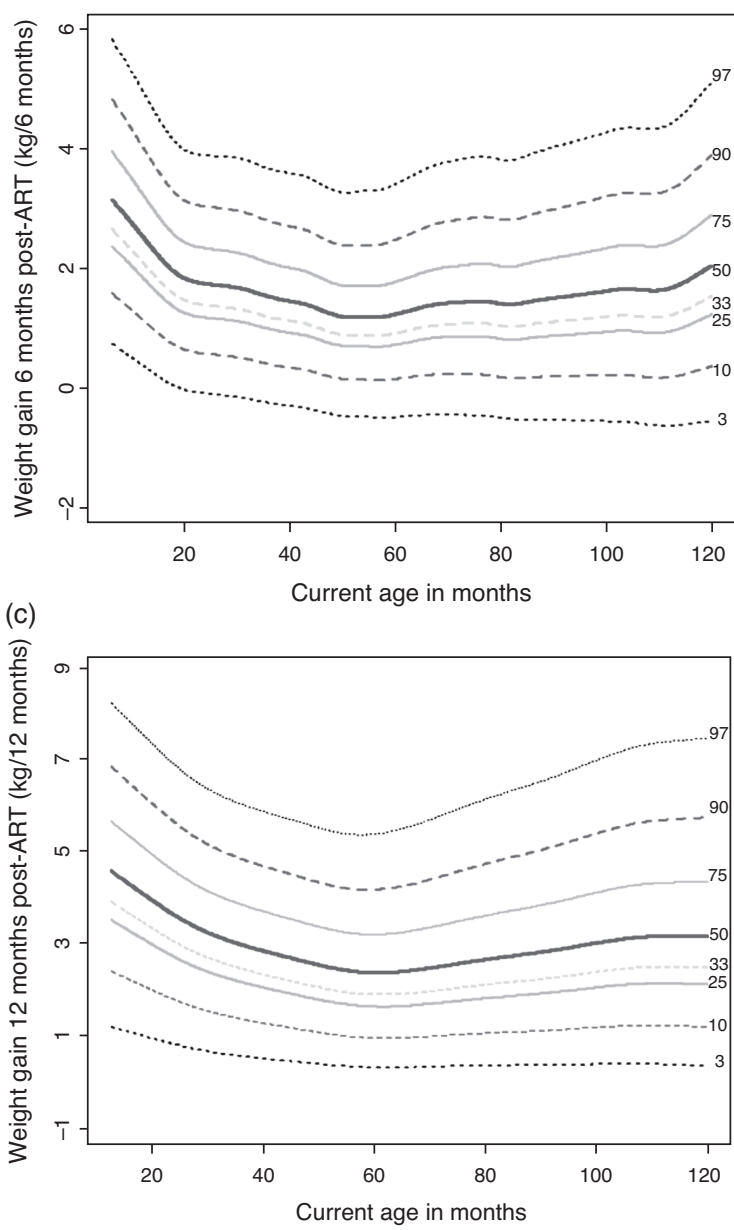

(b)

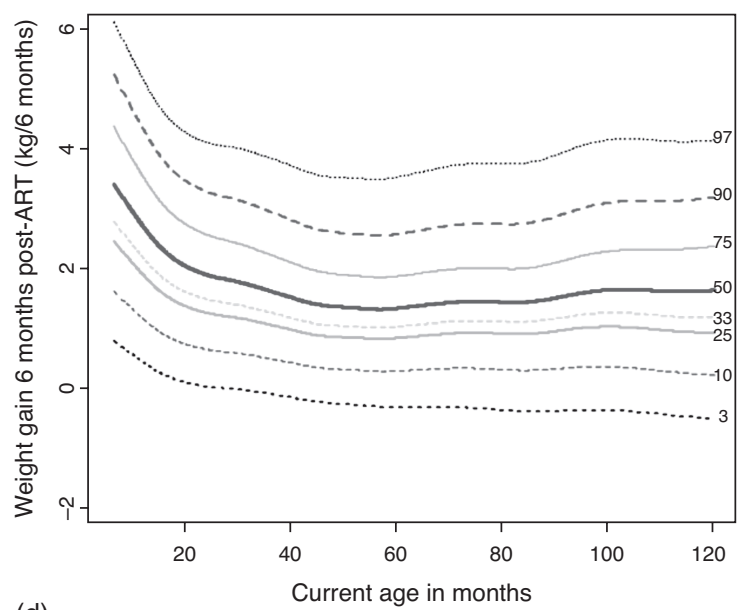

(d)

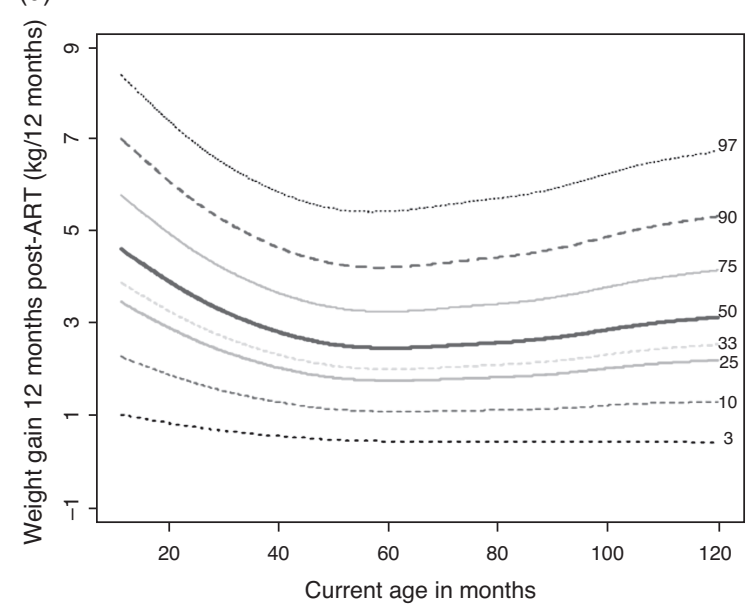

Fig. 1. Six-month and 12-month sex-specific and age-specific weight gain reference curves in children. (a) Centile curves for 6 months post-ART weight gain in females. (b) Centile curves for 6 months post-ART weight gain in males. (c) Centile curves for 12 months post-ART weight gain in females. (d) Centile curves for 12 months post-ART weight gain in males. Curves were obtained using Box Cox power exponential (BCPE) distribution and the generalized additive model for location, scale, and shape. Model for female at 6 months (a): BCPE (age, $\operatorname{df}(\mu)=12.9, \operatorname{df}(\sigma)=0.2, \operatorname{df}(v)=1, \operatorname{df}(\tau)=3)$. Model for males at 6 months $(b)$ : BCPE $($ age, $\mathrm{df}(\mu)=9.9, \operatorname{df}(\sigma)=0.2, \operatorname{df}(v)=2, \operatorname{df}(\tau)=2)$. Model for females at 12 months $(\mathrm{c})$ : BCPE $($ age, $\operatorname{df}(\mu)=4.8, \mathrm{df}(\sigma)=0, \mathrm{df}(v)=1, \mathrm{df}(\tau)=0)$. Model for males at 12 months $(\mathrm{d})$ : BCPE (age, $\mathrm{df}(\mu)=4.8, \mathrm{df}(\sigma)=0, \mathrm{df}(v)=1.3, \mathrm{df}(\tau)=0)$. df, degrees of freedom. 
(a)

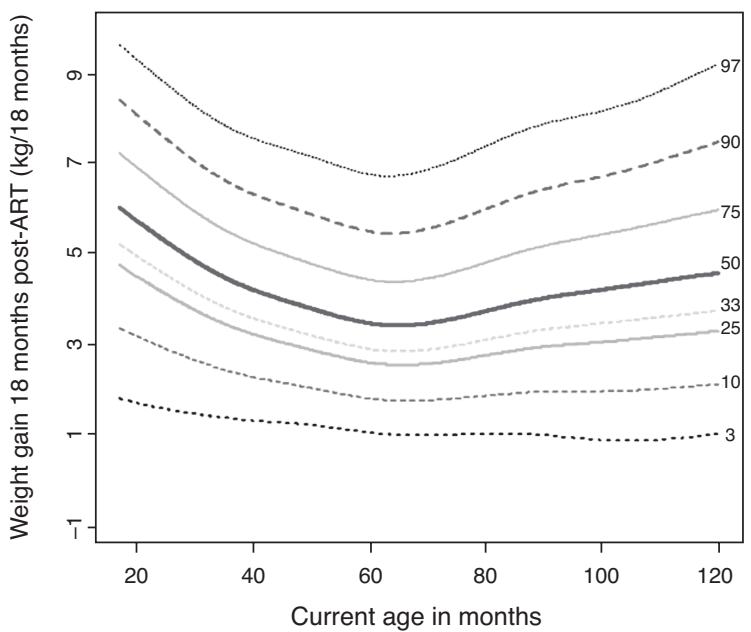

(c)

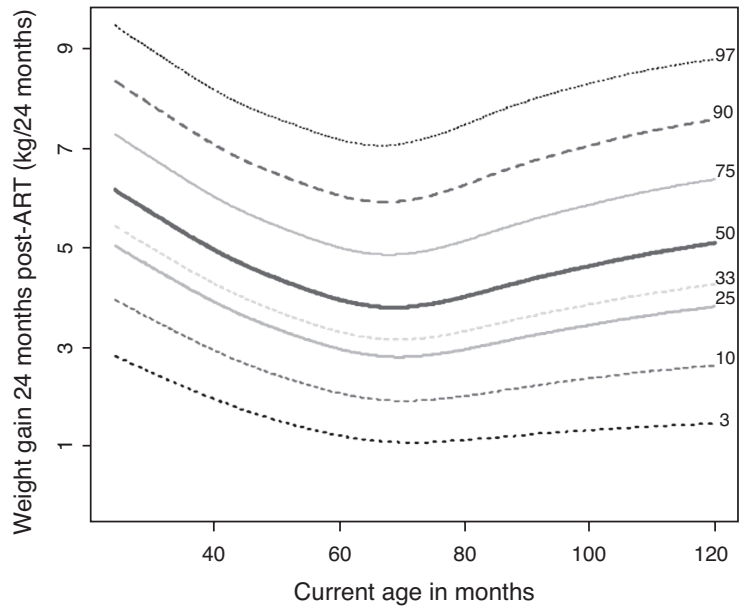

(b)

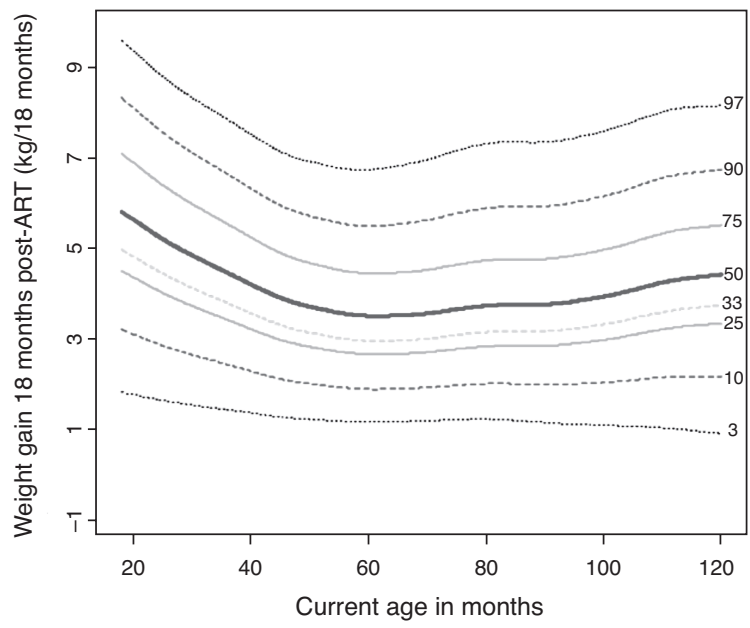

(d)

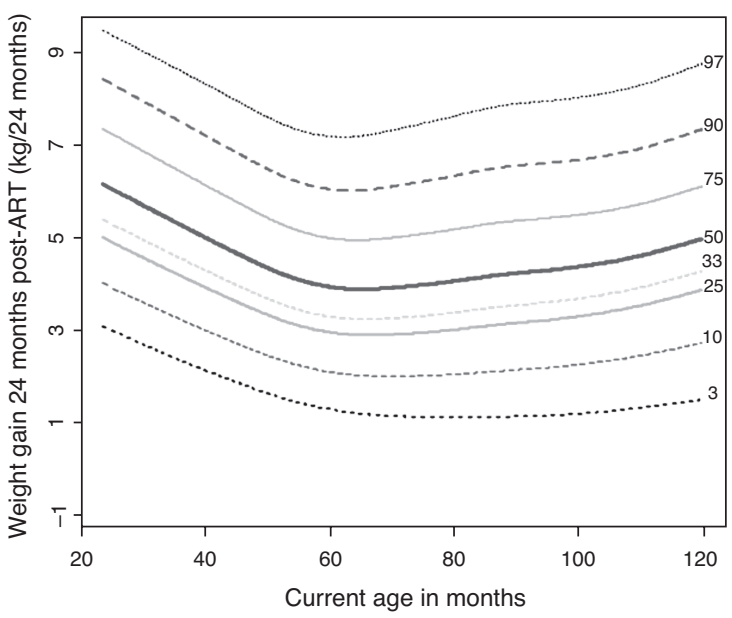

Fig. 2. Eighteen-month and 24-month sex-specific and age-specific weight gain reference curves in children. (a) Centile curves for 18 months post-ART weight gain in females. (b) Centile curves for 18 months post-ART weight gain in males. (c) Centile curves for 24 months post-ART weight gain in females. (d) Centile curves for 24 months post-ART weight gain in males. Curves were obtained using Box Cox power exponential (BCPE) distribution and the generalized additive model for location, scale, and shape. Model for females at 18 months (a): BCPE (age, $\mathrm{df}(\mu)=4.1, \mathrm{df}(\sigma)=0, \mathrm{df}(\boldsymbol{v})=3, \mathrm{df}(\tau)=0)$. Model for males at $18 \mathrm{months}(\mathrm{b})$ : BCPE (age, $\operatorname{df}(\mu)=6.1, \operatorname{df}(\sigma)=0, \operatorname{df}(v)=2, \operatorname{df}(\tau)=0)$. Model for females at 24 months (c): BCPE (age, $\operatorname{df}(\mu)=3, \operatorname{df}(\sigma)=1.5, \operatorname{df}(v)=0.8$, $\mathrm{df}(\tau)=0)$. Model for males at 24 months $(\mathrm{d})$ : BCPE (age, $\mathrm{df}(\mu)=3.8, \mathrm{df}(\sigma)=1.22, \mathrm{df}(v)=1, \mathrm{df}(\tau)=0)$. df, degrees of freedom.

those above was 2.97 (95\% CI: 2.03, 4.36) at 6 months and $2.28(95 \% \mathrm{CI}: 1.23,4.22)$ at 12 months. A doseresponse effect was observed for these associations with higher hazard ratios at lower weight gains, especially for the first 12 months of ART. For example, children with weight gains at the lowest (3rd) percentile had a nine-fold greater hazard of subsequent death compared with children with greater weight gain. The increased risk of death with lower weight gains persisted after 18 and 24 months of ART, but the estimates were imprecise due to the limited number of deaths that occurred after 18 months.

No statistical association was observed between the distribution of weight gain and time to virological suppression or time to virologic failure (Table 2).

\section{Discussion}

Data from recent randomized clinical trials in children [11] and in adults [10] show that routine laboratory monitoring for antiretroviral drug toxicity may not be needed in children and that $\mathrm{CD} 4^{+}$monitoring provides a small but significant reduction in disease progression or death after the second year on ART. In adults, despite results from a large multicountry cohort study showing that virological monitoring might have some added benefit [28], particularly after 2 years, results from a clinical trial shows that adding viral load to $\mathrm{CD}^{+}$monitoring provided no further benefits [10]. The trial in children identified monitoring weight gain as a sensitive indicator of first-line treatment failure [11]. 
Table 2. Association between lower percentile of weight gains at 6, 12, 18, and 24 months of antiretroviral therapy and time to mortality, virological suppression, and virological failure.

\begin{tabular}{|c|c|c|c|c|c|c|}
\hline & \multicolumn{2}{|c|}{ Time to death after 6 months } & \multicolumn{2}{|c|}{ Time to virological suppression ${ }^{a}$} & \multicolumn{2}{|c|}{ Time to virological failure ${ }^{b}$} \\
\hline & \multicolumn{2}{|c|}{ Hazard ratio $(95 \% \mathrm{Cl})$} & \multicolumn{2}{|c|}{ Hazard ratio $(95 \% \mathrm{Cl})$} & \multicolumn{2}{|c|}{ Hazard ratio $(95 \% \mathrm{Cl})$} \\
\hline & Crude & Adjusted $^{\mathrm{C}}$ & Crude & Adjusted $^{d}$ & Crude & Adjusted $^{\mathrm{e}}$ \\
\hline Percentile & \multicolumn{6}{|c|}{ Weight gain at 6 months after ART initiation } \\
\hline $3 \mathrm{rd}$ & $7.61(4.64,12.48)$ & $9.20(5.51,15.34)$ & $0.95(0.69,1.30)$ & $1.18(0.86,1.62)$ & $1.85(102,3.36)$ & $1.10(0.46,2.68)$ \\
\hline 10th & $6.86(4.62,10.20)$ & $8.61(5.74,12.92)$ & $1.01(0.83,1.21)$ & $1.11(0.92,1.34)$ & $1.50(1.02,2.21)$ & $0.76(0.43,1.34)$ \\
\hline 25th & $3.18(2.19,4.62)$ & $4.17(2.84,6.12)$ & $0.92(0.83,1.02)$ & $0.93(0.84,1.03)$ & $1.12(0.91,1.37)$ & $1.10(0.85,1.41)$ \\
\hline $33 \mathrm{rd}$ & $2.30(1.59,3.34)$ & $2.97(2.03,4.36)$ & $0.95(0.87,1.04)$ & $0.95(0.87,1.04)$ & $1.01(0.84,1.21)$ & $0.90(0.72,1.13)$ \\
\hline \multirow[t]{2}{*}{ 50th } & $1.45(0.99,2.12)$ & $1.80(1.22,2.66)$ & $0.93(0.86,1.01)$ & $0.91(0.84,0.99)$ & $0.98(0.83,1.15)$ & $0.95(0.78,1.16)$ \\
\hline & \multicolumn{6}{|c|}{ Weight gain at 12 months after ART initiation } \\
\hline $3 \mathrm{rd}$ & $5.96(2.65,13.40)$ & $7.33(3.20,16.78)$ & $1.07(0.81,1.40)$ & $1.11(0.86,1.46)$ & $1.21(0.67,2.21)$ & $0.92(0.45,1.91)$ \\
\hline 10th & $3.25(1.60,6.60)$ & $4.05(1.96,8.37)$ & $0.96(0.81,1.15)$ & $0.99(0.83,1.18)$ & $1.15(0.80,1.66)$ & $0.82(0.49,1.37)$ \\
\hline 25 th & $1.82(0.98,3.38)$ & $2.21(1.17,4.17)$ & $1.00(0.90,1.12)$ & $1.01(0.90,1.12)$ & $0.98(0.79,1.21)$ & $0.94(0.72,1.23)$ \\
\hline $33 \mathrm{rd}$ & $1.88(1.03,3.41)$ & $2.28(1.23,4.22)$ & $1.00(0.90,1.10)$ & $0.99(0.89,1.09)$ & $0.99(0.81,1.20)$ & $0.91(0.72,1.15)$ \\
\hline \multirow[t]{2}{*}{ 50th } & $1.39(0.76,2.54)$ & $1.64(0.88,3.04)$ & $0.97(0.88,1.07)$ & $0.95(0.86,1.04)$ & $0.92(0.77,1.10)$ & $0.90(0.72,1.11)$ \\
\hline & \multicolumn{6}{|c|}{ Weight gain at 18 months after ART initiation } \\
\hline $3 \mathrm{rd}$ & - & - & $0.94(0.70,1.25)$ & $1.11(0.74,1.67)$ & $1.00(0.52,1.93)$ & $0.61(0.22,1.68)$ \\
\hline 10th & $1.55(0.46,5.20)$ & $1.88(0.55,6.43)$ & $1.06(0.89,1.26)$ & $0.88(0.69,1.12)$ & $0.93(0.64,1.34)$ & $0.83(0.50,1.36)$ \\
\hline 25 th & $1.98(0.86,4.58)$ & $2.41(1.02,5.68)$ & $1.01(0.90,1.13)$ & $0.90(0.77,1.05)$ & $0.93(0.74,1.17)$ & $0.91(0.66,1.24)$ \\
\hline $33 \mathrm{rd}$ & $1.59(0.70,3.63)$ & $1.92(0.82,4.48)$ & $0.98(0.89,1.09)$ & $0.98(0.85,1.12)$ & $0.92(0.75,1.13)$ & $0.84(0.65,1.10)$ \\
\hline \multirow[t]{2}{*}{ 50th } & $1.90(0.81,4.49)$ & $2.32(0.97,5.58)$ & $0.94(0.85,1.03)$ & $0.93(0.81,1.06)$ & $0.99(0.81,1.19)$ & $0.96(0.76,1.21)$ \\
\hline & \multicolumn{6}{|c|}{ Weight gain at 24 months after ART initiation } \\
\hline $3 \mathrm{rd}$ & $2.76(0.35,21.53)$ & $3.34(0.42,26.34)$ & $0.75(0.54,1.02)$ & $0.79(0.52,1.21)$ & $1.31(0.78,2.21)$ & $1.21(0.56,2.64)$ \\
\hline 10th & $2.21(0.48,10.23)$ & $2.87(0.61,13.48)$ & $0.97(0.80,1.18)$ & $0.83(0.64,1.08)$ & $1.17(0.81,1.68)$ & $1.31(0.79,2.16)$ \\
\hline 25 th & $3.90(1.19,12.80)$ & $4.80(1.44,15.93)$ & $1.00(0.88,1.14)$ & $0.89(0.75,1.06)$ & $1.05(0.81,1.35)$ & $0.98(0.69,1.40)$ \\
\hline $33 \mathrm{rd}$ & $5.65(1.50,21.28)$ & $7.17(1.87,27.44)$ & $0.93(0.82,1.04)$ & $0.87(0.74,1.02)$ & $0.95(0.75,1.20)$ & $0.87(0.64,1.18)$ \\
\hline 50th & $4.79(1.03,22.15)$ & $5.99(1.28,28.08)$ & $0.93(0.83,1.04)$ & $0.92(0.79,1.02)$ & $0.90(0.73,1.11)$ & $0.89(0.68,1.18)$ \\
\hline
\end{tabular}

ART, antiretroviral therapy.

${ }^{a}$ Viral load $\leq 400$ HIV RNA copies/ml.

${ }^{b}$ Viral load measurement after at least 1 year of ART above 1000 copies $/ \mathrm{ml}$, two consecutive viral load measurements $>400 \mathrm{copies} / \mathrm{ml}$ after initial virologic suppression, or failure to achieve virological suppression after at least 1 year of ART.

${ }^{c}$ Adjusted for baseline weight-for-age $z$ scores (WAZ).

${ }^{\mathrm{d} A d j u s t e d}$ for baseline age, viral load, and region.

${ }^{\mathrm{e}}$ Adjusted for baseline age, viral load, and region.

Growth monitoring is routinely performed in the followup of children [26]. However, neither of the commonly used WHO and Fels normative references growth curves are valid for HIV-infected children starting ART [16]. This is mainly because the origin used for both of these curves is birth, although ART initiation in resourcelimited settings generally does not happen at birth. In this study, we were able to construct normative reference standards for weight gain at $6,12,18$, and 24 months of ART for HIV-infected children younger than 10 years. At 6 and 12 months on ART, the hazard of dying in children whose weight gain was below the $33^{\text {rd }}$ percentile was at least twice that of children who gained more weight. The strength of the association increased with decreasing weight gain.

We did not observe a correlation between weight gain and virological suppression or virological failure. This is contrary to findings from our single-clinic cohort study of South African children [16]. We speculate that it is because of selection bias, as viral load monitoring was not routinely available or accessible in most clinics and regions. In most regions outside of South Africa, children in routine care are only assessed by viral load in the presence of a clinical indication. As such, children with available viral load measurements are not representative of all children on ART.

The large sample size and extended follow-up allowed us to construct reference distributions through 24 months, and inclusion of five regions representing the regions of the world where virtually all pediatric HIV cases are found were important strengths of our study. Unfortunately, we did not have adequate and unbiased data on viral load and $\mathrm{CD}^{+}{ }^{+}$to assess the potential of monitoring weight gain alone or in combination with $\mathrm{CD}^{+}$as predictors of poor response to ART. Moreover, we had to limit the analysis to children 10 years or younger because of the high heterogeneity of weight gain after 10 years of age. [26] Finally, because of the open nature of the cohorts, occurrence of deaths and loss to follow up, numbers of children included in the analysis reduced with longer follow-up time points. The data thus need to be interpreted conditional on surviving and remaining on ART and in care to the time point of interest.

In conclusion, in areas with limited access to viral load or $\mathrm{CD}^{+}$measurement, monitoring weight gain post-ART using normative data developed specifically for HIVinfected children on ART could be a simple and highly 
valuable tool to identify those children at highest risk of death.

\section{Acknowledgements}

The authors acknowledge all of the children and their families followed up in the participating pediatric centers. The authors also thank the staff from all participating pediatric centers. The authors warmly thank all the investigators (see supplemental material for the full list) and pediatric coordinators from the Pediatric International Epidemiologic Databases to Evaluate AIDS (IeDEA) Regions contributing to the project: AsiaPacific (Annette Sohn), East Africa (Kara WoolsKaloustian), Southern Africa (M.A.D.), West Africa (Valériane Leroy), Central Africa (J.N. and Andrew Edmonds), and the IeDEA Pediatric Working Group: Melanie Bacon, Robin Huebner, Rosemary McKaig, Lynne Mofenson, Lori Schwarze.

M.Y., A.V.R., T.M., and F.B. designed the study. All authors helped with data collection. M.Y. analyzed data, M.Y. and A.V.R. wrote the first draft of the report. All authors contributed to the interpretation of the data and read and approved the final manuscript.

IeDEA is supported by the US National Institutes of Health's (NIH) National Institute of Allergy and Infectious Diseases, Eunice Kennedy Shriver National Institute of Child Health and Human Development and National Cancer Institute through grants to the below regions. The five regions which contributed data to this analysis are funded through grants U01AI069911 (East Africa), U01AI069924 (Southern Africa), U01AI069919 (West Africa), U01AI069907 (Asia-Pacific), and U01AI069927 (Central Africa). The TREAT Asia Pediatric HIV Observational Database is also supported by AIDS Life, Austria. T.M. is a recipient of NIH Fogarty International Center grants to the University of North Carolina and University of the Witwatersrand numbers U2RTW007370 and U2RTW007373. M.Y. is partially supported by the Central Africa IeDEA grant U01AI096299 and NIH R01HD075171. No funding bodies had any role in study design, data collection and analysis, decision to publish, or preparation of the manuscript.

\section{Conflicts of interest}

There are no conflicts of interest.

\section{References}

1. UNAIDS. Global report: UNAIDS report on the global AIDS epidemic 2013. Geneva, Switzerland: UNAIDS; 2013. http:// www.unaids.org/en/resources/campaigns/globalreport2013/ globalreport/ [Accessed 17 October 2014).
2. Newell ML, Coovadia H, Cortina-Borja M, Rollins N, Gaillard P, Dabis F. Mortality of infected and uninfected infants born to HIV-infected mothers in Africa: a pooled analysis. Lancet 2004; 364:1236-1243.

3. Bolton-Moore C, Mubiana-Mbewe M, Cantrell RA, Chintu N, Stringer EM, Chi BH. Clinical outcomes and CD4 cell response in children receiving antiretroviral therapy at primary healthcare facilities in Zambia. IAMA 2007; 298:1888-1899.

4. Stringer JS, Zulu I, Levy J, Stringer EM, Mwango A, Chi BH, et al. Rapid scale-up of antiretroviral therapy at primary care sites in Zambia: feasibility and early outcomes. JAMA 2006; 296:782793.

5. Sutcliffe CG, van Dijk JH, Bolton C, Persaud D, Moss WJ. Effectiveness of antiretroviral therapy among HIV-infected children in sub-Saharan Africa. Lancet Infect Dis 2008; 8:477-489.

6. Yotebieng M, Van Rie A, Luisiama J, Kitetele F, Edmonds A, Behets F. Survival, clinical, immunological and hematological outcomes of antiretroviral therapy among HIV-infected children attending a public clinic in Kinshasa, Democratic Republic of Congo. [Abstract]. 14th International Congress on Infectious Diseases (ICID), 12 march 2010, Miami, FL, USA.

7. Meyers TM, Yotebieng M, Kuhn L, Moultrie H. Antiretroviral therapy responses among children attending a large public clinic in Soweto, South Africa. Pediatr Infect Dis J 2011; 30: 974-979.

8. Panel on Antiretroviral Therapy and Medical Management of HIV-Infected Children. Guidelines for the use of antiretroviral agents in pediatric HIV infection; 2014: See http://aidsinfo.nih. gov/contentfiles/lvguidelines/pediatricguidelines.pdf [Accessed 17 October 2014].

9. Mermin J, Ekwaru JP, Were W, Degerman R, Bunnell $R$ Kaharuza $F$, et al. Utility of routine viral load, CD4 cell count, and clinical monitoring among adults with HIV receiving antiretroviral therapy in Uganda: randomised trial. $B M$ J 2011; 343:d6792.

10. Mugyenyi P, Walker AS, Hakim J, Munderi P, Gibb DM, Kityo F, et al. Routine versus clinically driven laboratory monitoring of HIV antiretroviral therapy in Africa (DART): a randomised noninferiority trial. Lancet 2010; 375:123-131.

11. Kekitiinwa A, Cook A, Nathoo K, Mugyenyi $P$, Nahirya-Ntege $P$, Bakeera-Kitaka $S$, et al. Routine versus clinically driven laboratory monitoring and first-line antiretroviral therapy strategies in African children with HIV (ARROW): a 5-year open-label randomised factorial trial. Lancet 2013; 381:1391-1403.

12. WHO. Consolidated guidelines on the use of antiretroviral drugs for treating and preventing HIV infection: recommendations for a public health approach June 2013. Geneva, Switzerland: World Health Organization; 2013: p 272.

13. WHO Multicentre Growth Reference Study Group. WHO Child Growth Standards: Growth velocity based on weight, length and head circumference: Methods and development. Geneva: World Health Organization; 2009: p. 242.

14. Baumgartner $\mathrm{RN}$, Roche $\mathrm{AF}$, Himes $\mathrm{JH}$. Incremental growth tables: supplementary to previously published charts. Am / Clin Nutr 1986; 43:711-722.

15. Roche AF, Himes JH. Incremental growth charts. Am J Clin Nutr 1980; 33:2041-2052.

16. Yotebieng M, Van Rie A, Moultrie H, Meyers T. Six-month gain in weight, height, and CD4 predict subsequent antiretroviral treatment responses in HIV-infected South African children. AIDS 2010; 24:139-146.

17. Edmonds A, Yotebieng M, Lusiama J, Matumona $\mathrm{Y}$, Kitetele $\mathrm{F}$ Napravnik S, et al. The effect of highly active antiretroviral therapy on the survival of HIV-infected children in a resourcedeprived setting: a cohort study. PLoS Med 2011; 8:e1001044.

18. Patel K, Hernan MA, Williams PL, Seeger JD, Mclntosh K, Van Dyke RB, Seage GR. Long-term effectiveness of highly active antiretroviral therapy on the survival of children and adolescents with HIV infection: a 10-year follow-up study. Clin Infect Dis 2008; 46:507-515.

19. Leroy V, Malateste K, Rabie H, Lumbiganon P, Ayaya S, Dicko F, et al. Outcomes of antiretroviral therapy in children in Asia and Africa: a comparative analysis of the IeDEA pediatric multiregional collaboration. I Acquir Immune Defic Syndr 2013; 62:208-219.

20. leDEA Pediatric Working Group. A survey of paediatric, HIV. programmatic and clinical management practices in Asia and sub-Saharan Africa: the International epidemiologic Databases to Evaluate, AIDS. (IeDEA). J Int AIDS Soc 2013; 16:17998. 
21. Carey VJ, Yong FH, Frenkel LM, McKinney RM. Growth velocity assessment in paediatric AIDS: smoothing, penalized quantile regression and the definition of growth failure. Stat Med 2004; 23:509-526.

22. Rigby RA, Stasinopoulos DM. Generalized additive models for location, scale and shape. I Royal Statist Soci Series C (Appl Statist) 2005; 54:507-554.

23. Rigby RA, Stasinopoulos DM. Smooth centile curves for skew and kurtotic data modelled using the Box-Cox power exponential distribution. Stat Med 2004; 23:3053-3076.

24. Royston P, Wright EM. Goodness-of-fit statistics for agespecific reference intervals. Stat Med 2000; 19:2943-2962.

25. van Buuren S, Fredriks $M$. Worm plot: a simple diagnostic device for modelling growth reference curves. Stat Med 2001; 20:1259-1277.
26. WHO Multicentre Growth Reference Study Group. WHO child growth standards: length/height-for-age, weight-for-age, weightfor-length, weight-for-height, and body mass index-for-agemethods and development. Geneva, Switzerland: WHO Press; 2006: http://apps.who.int/iris/bitstream/10665/43413/1/ 924154693X_eng.pdf [Accessed 17 October 2014].

27. Lin DY, Wei LJ, Ying Z. Checking the Cox model with cumulative sums of martingale-based residuals. Biometrika 1993; 80: 557-572.

28. Estill J, Egger M, Johnson LF, Gsponer T, Wandeler G, et al. Monitoring of antiretroviral therapy and mortality in HIV programmes in Malawi, South Africa and Zambia: mathematical modelling study. PLOS One 2013; 8:e57611. 